\title{
Beyond Acute Respiratory Distress: Multiple Organ Effects and Early Rehabilitation in COVID-19
}

\author{
Elizabeth PAPATHANASSOGLOU, RN, MSc, PhD, ${ }^{a} \odot$ Kiran POHAR MANHAS, JD, PhD, ${ }^{b}$ Elizabeth \\ KUSI-APPIAH, RN, MN"
}

The Novel Severe Acute Respiratory Syndrome Coronavirus 2 (SARS-COV-2) coronavirus has caused rapidly spreading disease (COVID-19) worldwide, accounting for an unprecedented increase in intensive care unit (ICU) admissions (Phua et al., 2020), and tens of thousands of deaths globally. The Surviving Sepsis Campaign COVID-19 panel released comprehensive clinical guidance for the management of affected patients in the ICU (Alhazzani et al., 2020); while new recommendations are formulated addressing specific contexts (Phua et al., 2020). Two important aspects of the SARS-CoV-2 infection have emerged since the beginning of the pandemic, which have significant implications for critical care nursing. First, apart from the lungs, SARS-CoV-2 can directly affect multiple organs and tissues, which may be a factor in the high mortality observed worldwide. Secondly, the extensive respiratory, neuromuscular, cognitive, and even psychiatric manifestations of COVID-19 call for early rehabilitation efforts, starting while patients are in the acute phase of hospitalization.

Severe COVID-19 seems to have two discernible phases. The acute phase most often encompasses an acute respiratory distress or other organ dysfunction with acute decompensation. This is followed by a convalescent phase at which the long-term effects of critical illness, as well as those of extrapulmonary disease, may become apparent. The post-ICU syndrome (PICS), encompassing impairments in organ system functions, cognition, mental health, and physical function (Ohtake et al., 2018), is a well-recognized group of sequelae affecting the quality of life and recovery of survivors of critical illness. In the case of COVID-19, some of those impairments may be due to viral invasion of multiple tissues, and not just the aftermath of critical illness. In this article, we present a brief evidence-based summary of the multiple organ manifestations of COVID-19, followed by implications for rehabilitation at the acute and postacute phase.

\section{MULTIPLE ORGAN EFFECTS OF COVID-19}

SARS-CoV-2 seems to employ at least two mechanisms that facilitate infection of many cell types, and evasion of the host's immune defenses. It enters the cell by binding angiotensin-converting enzyme 2 (ACE2), which is broadly expressed in multiple tissues including the gastrointestinal tract, testes, kidneys, heart, lungs, liver, adrenal and thyroid gland, blood vessels, brain, bone marrow, adipose tissue, and muscle ( $\mathrm{Li}, \mathrm{Li}$, Zhang, \& Wang, 2020). Its spike protein, which facilitates receptor binding, remains "hidden" until after binding (Shang et al., 2020).

\section{Effects on Organ Systems}

In the lungs, SARS-CoV-2 causes diffuse alveolar damage, airway inflammation, focal hemorrhage, and fibrosis (Barton, Duval, Stroberg, Ghosh, \& Mukhopadhyay, 2020; Brugliera et al., 2020), which manifest as severe respiratory distress and prolonged respiratory disease. In

\footnotetext{
${ }^{a}$ University of Alberta, Edmonton, Alberta, Canada. E-mail: papathan@ualberta.ca

${ }^{b}$ Neurosciences Rehabilitation \& Vision Strategic Clinical Network, Alberta Health Services, Calgary, Alberta, Canada.

${ }^{c}$ University of Alberta, 5-262 Edmonton Clinic Health Academy, Edmonton, Alberta, Canada
} 
the heart, infection may cause cardiomyocyte death, lymphocytic infiltration, vasculitis, and thrombosis, manifesting as myocardial injury, possibly with pain and ST elevation, cardiomyopathy or arrhythmias (Akbarshakh \& Eduardo, 2020). Cardiac manifestations seem to ensue a few days after the onset of respiratory disease (Akbarshakh \& Eduardo, 2020). Similarly, cell degeneration, microthrombi, and lymphocytic infiltration are observed in the liver and kidney (Brugliera et al., 2020). The incidence of liver injury may be up to $53 \%$, mainly manifesting as altered ALT/AST levels (Xu, Liu, $\mathrm{Lu}$, Yang, \& Zheng, 2020). It is not clear to which degree liver injury is also induced by drugs, as this is a side effect of antivirals and steroids. Although SARS-CoV-2 directly invades kidney tissues, hypoxia and coagulopathy may also have synergistic effects in acute kidney injury ( $\mathrm{Su}$ et al., 2020). Gastrointestinal symptoms, including diarrhea and vomiting, are common especially in children, even in the absence of respiratory symptoms. The virus invades gastrointestinal epithelial cells, and is excreted in the feces, possibly persisting as long as 11 days after sputum excretion (Tian, Rong, Nian, \& He, 2020).

\section{Effects on the Vascular and Immune System}

The endothelium has ample ACE-2 receptors, which take up the virus. Disseminated intravascular coagulation (DIC) may develop in a few days in COVID-19 patients, mediated by both sepsis and endothelial infection (Harenberg \& Favaloro, 2020). The spleen is also affected, and T-cell numbers are suppressed (Brugliera et al., 2020).

\section{Effects on the Central and Peripheral Nervous System}

Invasion of the peripheral and central nervous systems may develop with hematogenous spread. Brain edema, anosmia, dysgeusia, ataxia, and consciousness alterations have been documented (Baig, Khaleeq, Ali, \& Syeda, 2020). Peripheral myopathy and neuropathy may ensue as either a side effect of prolonged critical illness and/or a result of neuroinvasion (Mao et al., 2020). Some patients may present with cerebrovascular accidents, potentially due to vascular complications (Avula et al., 2020). Up to half of patients with severe disease may have neurologic involvement (Mao et al., 2020).

\section{Effects on Cognitive Functions and Mental Health}

Data on the potential psychiatric effects of COVID19 are emerging (Ramaswamy, 2020). Up to 70\% of severely ill COVID-19 patients may develop delirium, probably due to the recognized effects of critical illness, neurological invasion, and hypoxia (O’Hanlon \& Inouye, 2020). Additionally, isolation precautions, isolation from family, and limited contact with staff who don protective equipment may perpetuate the anxious ideation of delirium (O’Hanlon \& Inouye, 2020).

\section{EARLY REHABILITATION NEEDS}

Upstream strategies are needed to offset respiratory, pulmonary, and neuromuscular complications characterizing COVID-19, especially for patients with advanced age, obesity, comorbidities, and organ failure (Brugliera et al., 2020; Landry et al., 2020).

\section{Early Respiratory Rehabilitation}

In the acute phase of SARS-CoV-2 infection, clinical parameters such as body temperature, respiratory rate, and oxygen saturation could indicate the type and intensity of respiratory rehabilitation needed (Kiekens et al., 2020). In cases where invasive intubation is used, weaning from ventilation and intubation may be followed by several days of clinical instability (Kiekens et al., 2020). Postural management of patients with bed rest angled at $45^{\circ}-60^{\circ}$ could promote ventilation if tolerated by the patient's clinical condition (Brugliera et al., 2020). The febrility of SARS-CoV-2 infection and demand for isolation which restricts movement creates a perfect condition for immobilization syndrome and ineffective sputum drainage (Brugliera et al., 2020). In hypersecretive patients with good breathing, bronchial clearance techniques such as controlled 
deep coughing, controlled huff coughing, and chest percussions could facilitate mucus drainage (Brugliera et al., 2020).

\section{Early Neuromuscular Rehabilitation}

An interdisciplinary approach using physiotherapy could progress patients' recovery, reduce the length of hospitalization, and facilitate patients' reintegration into the community (Landry et al., 2020). The goal for rehabilitation in the acute phase is to build patients' capacity for thorough rehabilitation after the infectious phase. Patient weakness or instability, as well as a focus on life preservation, in the critical-care-focused acute phase limit the provision of rehabilitation in the acute care context (Borg \& Stam, 2020). Emerging data show that prolonged prone ventilation, as used in most COVID-19 patients, is associated with later complications including muscle weakness, reduced joint mobility, balance, postural disorders, and impairment in activities of daily living (ADL) (Kiekens et al., 2020). When respiratory, hemodynamic, and cognitive stability is established, early mobility measures can be initiated. Passive range of motion exercises can attenuate deconditioning associated with prolonged immobilization (Woten \& Caple, 2018). As per the patient's condition, mobility exercises could start with in-bed exercises, such as lateral rolling and tilt-table exercises (Brummel et al., 2012; Woten \& Richards, 2018). Patients who show positive signs for mobility readiness can be supported to perform out-of-bed activities, such as standing and reaching in and out of the base of support (Woten \& Richards, 2018).

Neuromuscular damage from prolonged prone immobilization and invasive ventilation can cause dysphagia (Kiekens et al., 2020). Following extubation, patients' swallowing reflexes must be assessed. Weaning patients of enteral feeding should be gradual until swallowing function is restored (Brugliera et al., 2020). Simulated training in $\mathrm{ADL}$ for patients with diminished function is essential for the restoration of functional independence (Brummel et al., 2012).
Sleep disturbances may arise from metabolic abnormalities, disease processes, and the ICU environment. Apart from nonpharmacological measures as described in current guidelines (Devlin et al., 2018), melatonin has demonstrated safety in stabilizing the sleep-wake cycle with its immune-enhancing features, anti-inflammatory, and anti-oxidative effect as an additional benefit (Zambrelli, Canevini, Gambini, \& D'Agostino, 2020).

\section{Cognitive and Psychosocial Rehabilitation}

Anxiety from ICU hospitalization and anoxic damage from the disease process can lead to cognitive decline. Cognitive engagement strategies using progressively challenging exercises on orientation, attention, and memory could improve cognitive functioning (Woten \& Richards, 2018). Engaging patients in the discussions of topics of their interest and hobbies in the early phase of convalescence enhances patient-centeredness (Brummel et al., 2012).

Depression and anxiety have been noted in most COVID-19 patients, but it is unclear whether these are owed to the disease process or the trajectory of care (Troyer, Kohna, \& Hong, 2020). A seemingly attributable factor is the restriction in family visitation, which increases symptom burden (Ahmed et al., 2020). Encouraging videoconference and regular phone communication between patients and significant others can support patients' mental health (Kiekens et al., 2020).

Overall, maintaining a balance between patients' rehabilitation needs and the risk of SARS-CoV-2 spread remains a challenge. While care providers risk exposure to aerosols and secretions from patients during rehabilitation interventions, the shortage of appropriate personal protective equipment increases the risk of SARS-CoV-2 spread to and through care providers (Bartolo et al., 2020).

\section{POST-ICU REHABILITATION}

When the critically ill COVID-19 patient stabilizes sufficiently to move to inpatient care and 
eventually discharge, the post-ICU rehabilitation considerations must be ramped up accordingly. There are long-term implications of SARS-CoV2 infection itself, and from the experience of severe respiratory illness and the secondary disabilities of ICU treatments, including critical illness polyneuropathy (CIP) and critical illness myopathy (CIM), both as part of PICS (Kiekens et al., 2020; Stam, Stucki, \& Bickenbach, 2020). The longer the patient stays in the ICU, the higher the risk for long-term physical, cognitive, and psychiatric complications (Stam et al., 2020). CIP and CIM syndromes affect approximately $25 \%-45 \%$ of critically ill patients during and after ICU stays involving mechanical ventilation, which lead to severe neurodegenerative complications including flaccid and symmetric paralysis, limb and respiratory muscle weakness, systemic inflammatory response syndrome, or multiple organ failure (Stam et al., 2020).

The consequences of PICS are personally and socially disruptive (Stam et al., 2020). Nearly onethird of patients who experience PICS cannot return to work, and another third do not return to their pre-ICU employment (or a position with a pre-ICU salary) (Stam et al., 2020). Patients experiencing CIP experience decreased exercise capacity, disability, and compromised quality of life for months, even years, after discharge from the ICU (Stam et al., 2020). Further COVID-19 sequelae that require rehabilitation in the postICU phase include dysphagia, muscle weakness, CIP, CIM, reduced joint mobility, neck and shoulder pain (due to prolonged proning), difficulty in verticalization, impaired balance and gait, limitations in the ADL, difficulty awakening with long-lasting confusional state, and psychological issues (Kiekens et al., 2020).

The focus of rehabilitation post-ICU mirrors the nature of concerns in the ICU, but with increased breadth and intensity. Both postacute rehabilitation assessment and treatment could be synchronous (in real-time, whether in-person or via telehealth) or asynchronous (e.g., prerecorded, customized exercise plan) (Sheehy, 2020). Postacute respiratory rehabilitation may include inspiratory muscle training (if inspiratory muscles are weak); deep, slow breathing; thoracic expression; diaphragmatic breathing; mobilization of respiratory muscles; airway clearance techniques, and positive expiratory pressure devices (Sheehy, 2020). Mobility and functional rehabilitation should be assessed and redressed (Thornton, 2020). Assessment could include muscle joint range of motion, strength testing, balance (using Berg Balance Scale), and exercise capacity testing (using the 6-minute walk test and cardiopulmonary exercise testing). Physiotherapy following early mobilization initiated in the ICU may include active limb exercises, progressive muscle strengthening, neuromuscular electrical stimulation, and aerobic reconditioning (Sheehy, 2020). Occupational therapy would focus on ADLs and targeted cognitive interventions to facilitate functional independence (Sheehy, 2020). Psychological interventions would target patient needs around depression, anxiety, or PTSD (Brugliera et al., 2020).

Multidisciplinary care is recommended for postICU rehabilitation for COVID-19 survivors. Inpatient rehabilitation could be provided by physiotherapists, occupational therapists, speechlanguage pathologists, and psychologists or social workers (Sheehy, 2020). At discharge, rehabilitation needs continue, and the healthcare team expands. Some COVID-19 patients will require discharge to specialty tertiary rehabilitation centers, while others may be discharged to the community-based care of their primary care healthcare team (Thornton, 2020). Post-ICU rehabilitation will require communication and clarity between ICU and inpatient teams, and between acute and community care teams, to ensure appropriate discharge planning (Thornton, 2020). These multidisciplinary teams may include physiatry, primary care, physical therapy, occupational therapy, critical care, psychology, and speech-language pathology (Kiekens et al., 2020; Stam et al., 2020). 


\section{CONCLUSION}

As critical care professionals devise new ways to improve COVID-19 patients' outcomes under conditions that often test the limits of both healthcare systems and healthcare workers, an additional challenge needs to be addressed: the increased needs for early and post-ICU rehabilitation. In this article, we briefly described multiple organ involvement, and delineated a crude framework for early rehabilitation efforts starting while COVID-19 patients are still in the ICU and continuing after ICU discharge.

\section{REFERENCES}

Ahmed, H., Patel, K., Greenwood, D., Halpin, S., Lewthwaite, P., Salawu, A., . . . Sivan, M. (2020). Long-term clinical outcomes in survivors of coronavirus outbreaks after hospitalization or ICU admission: A systematic review and meta-analysis of follow-up studies. doi:10.1101/2020.04.16.20067975

Akbarshakh, A., \& Eduardo, M. (2020). COVID-19 and the heart. Circulation Research, 1O(16), 1443-1455. doi:10.1161/CIRCRESAHA.120.3 17055

Alhazzani, W. M., Morten, H., Arabi, Y. M., Loeb, M., Gong, M. N., Fan, E., . . . Rhodes, A. (2020). Surviving sepsis campaign: Guidelines on the management of critically ill adults with Coronavirus disease 2019 (COVID-19). Critical Care Medicine, 48(6), 440-469. doi: 10.1097/CCM.0000000000004363

Avula, A., Nalleballe, K., Narula, N., Sapozhnikov, S., Dandu, V., Toom, S., . . Elsayegh, D. (2020). COVID-19 presenting as stroke. Brain Behavior and Immunity. 1-5.doi:10.1016/j.bb i.2020.04.077

Baig, A. M., Khaleeq, A., Ali, U., \& Syeda, H. (2020). Updates on what ACS reported: Emerging evidences of COVID-19 with nervous system involvement. ACS Chemical Neuroscience, 11(9), 1204-1205. doi:10.1021/acs chemneuro.0c00181

Bartolo, M., Intiso, D., Lentino, C., Sandrini, G., Paolucci, S., \& Zampolini, M. (2020). Urgent measures for the containment of the Covid-19 epidemic in the Neurorehabilitation/
Rehabilitation Departments in the phase of maximum expansion of the epidemic (Governing Council of the Italian Society of Neurological Rehabilitation SIRN) Report. Bartolo et al's article is Frontiers in Neurology, abbreviated as Front. Neurol. URL: https://doi.org/10.3389/fneur.2020.00423.

Barton, L. M., Duval, E. J., Stroberg, E., Ghosh, S., \& Mukhopadhyay, S. (2020). COVID-19 autopsies. American Journal of Clinical Pathology, 153(6), 725-733. doi:10.1093/ajcp/aqaa062

Borg, K., \& Stam, H. (2020). Editorial: Covid-19 and physical and rehabilitation medicine. Journal of Rehabilitation Medicine, 52(4), jrm00045. doi:10.2340/16501977-267 9. Retrieved from https://web-b-ebscohost-co m.ahs.idm.oclc.org/ehost/pdfviewer/pdfview er?vid=1\&sid=ce86ec77-d5f4-496b-b03e-99d4 c2c487d7\%40pdc-v-sessmgr03

Brugliera, L., Spina, A., Castellazzi, P., Cimino, P., Tettamanti, A., Houdayer, E., . . . Iannaccone, S. (2020). Rehabilitation of Covid-19 patients. Journal of Rehabilitation Medicine, 52(4), 13. doi:10.2340/16501977-2678

Brummel, N. E., Jackson, J. C., Girard, T. D., Pandharipande, P. P., Schiro, E., Work, B., . . . Ely, E. W. (2012). A combined early cognitive and physical rehabilitation program for people who are critically ill: The activity and cognitive therapy in the intensive care unit (ACTICU) trial. Physical Therapy, 92(12), 15801592. doi:10.2522/ptj.20110414

Devlin J., Gelinas C., Needham D., Slooter A.I, Pandharipande P …Alhazzani W.(2018). Clinical practice guidelines for the prevention and management of pain, agitation/sedation, delirium, immobility, and sleep disruption in adult patients in the ICU. Critical Care Medicine, 46(9), e825-e873, doi:10.1097/CCM.0000000000003299

Harenberg, J., \& Favaloro, E. (2020). COVID19: Progression of disease and intravascular coagulation - present status and future perspectives. Clinical Chemistry and Laboratory Medicine, 1-8. doi:10.1515/cclm-2020-0502

Kiekens, C., Boldrini, P., Andreoli, A., Avesani, R., Gamna, F., Grandi, M., . . . Negrini, S. 
(2020). Rehabilitation and respiratory management in the acute and early post-acute phase. "Instant paper from the field" on rehabilitation answers to the Covid-19 emergency. European Journal of Physical and Rehabilitation Medicine. doi:10.23736/S1973-9087.20. 06305-4

Landry, M. D., Tupetz, A., Jalovcic, D., Sheppard, P., Jesus, T. S., \& Raman, S. R. (2020). The novel coronavirus (COVID-19): Making a connection between infectious disease outbreaks and rehabilitation. Advance online publication. Physiotherapy Canada. doi:10.3138/ptc2020-0019

Li, M., Li, L., Zhang, Y., \& Wang, X. S. (2020). Expression of the SARS-CoV-2 cell receptor gene ACE2 in a wide variety of human tissues. Infectious Diseases of Poverty, 9(45), 17. doi:10.1186/s40249-020-00662-x

Mao L., Jin H., Wang M., Hu Y., Chen S., He Q ... Hu B. (2020). Neurologic manifestations of hospitalized patients with Coronavirus Disease 2019 in Wuhan, China.JAMA Neurol ,77(6), 683-690. doi: 10.1001/jamaneurol.2020.1127

O’Hanlon, S., \& Inouye, S. K. (2020). Delirium: A missing piece in the COVID-19 pandemic puzzle. Age and Ageing 1-2. doi:10.1093/agei ng/afaa094

Ohtake, P. J., Lee, A. C., Scott, J. C., Hinman, S. R., Ali, N. A., Hinkson, C. R., . . . Spires, C. M. (2018). Physical impairments associated with post-intensive care syndrome: Systematic review based on the World Health Organization's international classification of functioning, disability, and health framework. Physical Therapy, 98(8), 631-645. doi:10.109 3/ptj/pzy059

Phua, J., Weng, L., Ling, L., Egi, M., Lim, C. M., Divatia, V., . . . Du, B. (2020). Intensive care management of coronavirus disease 2019 (COVID-19): Challenges and recommendations. The Lancet Respiratory Medicine, 8(5), 506-517. doi:10.1016/S2213-2600(20) 30161-2

Ramaswamy, D. (2020). Coronavirus and mental illnesses: Is COVID-19 linked to psychosis? International Business Times (USA). Retrieved from https://www.ibtimes.c om/coronavirus-mental-illnesses-covid-19-lin ked-psychosis-2973937

Shang, J., Wan, Y., Luo, C., Ye, G., Geng, Q., Auerbach, A., \& Li, F. (2020). Cell entry mechanisms of SARS-CoV-2. Proceedings of the National Academy of Sciences of the United States of America, 17(21): 11727-11734. doi:1 $0.1073 /$ pnas.2003138117

Sheehy, L. M. (2020). Considerations for postacute rehabilitation for survivors of COVID19. JMIR Public Health and Surveillance, 6(2), e19462. doi:10.2196/19462. Retrieved from https://publichealth.jmir.org/2020/2/ e19462/

Stam, H. J., Stucki, G., \& Bickenbach, J. (2020). Covid-19 and post intensive care syndrome: A call to action. Journal of Rehabilitation Medicine, 52(4), jrm0004. doi:10.2340/16501 977-2677. Retrieved from https://web-b-ebsco host-com.ahs.idm.oclc.org/ehost/pdfviewer/p dfviewer?vid=1\&sid=a901e031-8940-486d-a7 09-d3d8243a3136\%40sessionmgr 103

Su, H., Yang, M., Wan, C., Yi, L. X., Tang, F., Zhu, H. Y., . . . Zhang, C. (2020). Renal histopathological analysis of 26 postmortem findings of patients with COVID-19 in China. Kidney International 1-9. doi:10.1016/j.kint.2020. 04.003

Tian, Y., Rong, L., Nian, W., \& He, Y. (2020). Review article: Gastrointestinal features in COVID-19 and the possibility of faecal transmission. Alimentary Pharmacology and Therapeutics, 51(9), 843-851. doi:10.1111/ apt.15731

Thornton, J. (2020). Covid-19: The challenge of patient rehabilitation after intensive care. British Medical Journal, 369, m1787. doi:10. 1136/bmj.m1787

Troyer, E. A., Kohna, J. N., \& Hong, S. (2020). Are we facing a crashing wave of neuropsychiatric sequelae of COVID-19? Neuropsychiatric symptoms and potential immunologic mechanisms. Brain, Behavior, and Immunity.1-6.doi:10.1016/j.bbi. 2020.04.027 
Woten M.R.B,Caple CRBM. Editorial: Early Mobilization of Patients in the Intensive Care Unit (ICU). CINAHL Nursing Guide [Internet]. Retrieved from:https://search-ebscohost-com .login.ezproxy.library.ualberta.ca/login.aspx ?direct=true $\& \mathrm{db}=$ nup $\& \mathrm{AN}=\mathrm{T} 708324 \&$ site $=$ ed s-live\&scope $=$ site.

Xu, L., Liu, J., Lu, M., Yang, D., \& Zheng, X. (2020). Liver injury during highly pathogenic human coronavirus infections. Liver International, 4O(5), 998-1004. doi:10.1111/ liv. 14435

Zambrelli, E., Canevini, M., Gambini, O., \& D'Agostino, A. (2020). Delirium and sleep disturbances in COVID19: A possible role for melatonin in hospitalized patients? Sleep Medicine, 7O, 111. doi:10.1016/j.sleep.2020. 04.006 